\title{
A Dynamic Hysteresis Model for THUNDER Transducers
}

\author{
Brian L. Ball ${ }^{1}$, Ralph C. Smith ${ }^{2}$ and Zoubeida Ounaies ${ }^{3}$ \\ ${ }^{1,2}$ Center for Research in Scientific Computation, North Carolina State Univ., Raleigh, NC 27695 \\ ${ }^{3}$ Mechanical Engineering, Virginia Commonwealth University, Richmond, VA 23284-3015
}

\begin{abstract}
This paper summarizes a nonlinear model which quantifies the displacements generated in THUNDER actuators in response to applied voltages for a variety of boundary conditions and exogenous loads. A PDE model is constructed using Newtonian principles to quantify the displacements in the actuator due to field inputs to the piezoceramic patch. A free energy based hysteretic stress-strain relation is employed to model hysteresis inherent to the PZT. A finite element method and Crank-Nicholson scheme are developed to discretize the model; properties of the model are illustrated through comparison with experimental data.
\end{abstract}

Keywords: THUNDER actuators, displacement model, hysteresis, piezoceramic, transition element, polarization, neutral surface, thin shell.

\section{Introduction}

THUNDER actuators, as depicted in Figure 1, offer the capability for generating large strains and forces due to a variety of mechanisms including improved robustness through the manufacturing process and increased electromechanical coupling due to their inherent shape. In this paper, we discuss modeling techniques for quantifying the displacements generated by THUNDER actuators in response to applied voltages for a variety of boundary conditions. The development of numerical methods is also addressed and the accuracy of the resulting finite dimensional models is illustrated through comparison with experimental data.

Model development is considered in two steps: (i) a constitutive relation is derived through characterization of the hysteresis and nonlinear saturation in the PZT and (ii) A PDE model is developed for the actuator behavior based on Newtonian principles. The first component is based on a free energy model for hysteresis in ferroelectric materials as outlined in [12] and only those details necessary for the development of the subsequent PDE model will be discussed. As detailed in [4], the characteristic curved shape of THUNDER actuators is due primarily to differing thermal coefficients in the constituent materials, which produce thermal stresses in the actuator during cooling, and secondarily to the reorientation of dipoles during repoling. We note that the quantification of strains due to thermal gradients has been investigated for a variety of applications (e.g., see $[2,5,6,7])$ with certain aspects having been considered for THUNDER actuators $[4,8]$. The quantification of stresses and strains due to repoling is based on domain theory for general ferroelectric materials $[9,10,14,15]$. Thin shell theory is then employed to develop PDE models which quantify the stresses and displacements throughout the actuator when voltage is applied to the piezoceramic patch.

Because the PDE model is infinite dimensional, approximation techniques must be considered to obtain a finite dimensional model which is appropriate for implementation. This is accomplished through a Galerkin finite element approach utilizing cubic Hermite elements in addition to transition elements to achieve the appropriate continuity across material interfaces. This numerical approach differs from that used in [3], where a linear and

\footnotetext{
${ }^{1}$ Email: blball@unity.ncsu.edu, Telephone: (919) 332-7957

${ }^{2}$ Email: rsmith@eos.ncsu.edu, Telephone: (919) 515-7552

${ }^{3}$ Email: zounaies@vcu.edu, Telephone: (804) 827-7025
} 


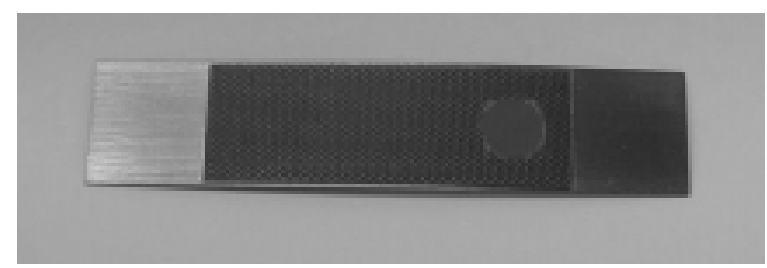

Figure 1. A THUNDER actuator manufactured by FACE International corporation.

cubic Hermite model was employed, in that the finite element method in this paper significantly reduces the order of the model while achieving higher numerical accuracy.

The geometry of the THUNDER actuators is outlined in Section 2 and a model which quantifies the hysteretic electric field-polarization relation is summarized in Section 3. The PDE model quantifying the displacements is then presented in Section 4 along with boundary conditions which characterize a variety of experimental setups. The numerical methods are discussed in Section 5 and examples illustrating the performance of the resulting finite dimensional model are presented in Section 6. Finally, extensions and future work is outlined in Section 7.

\section{Actuator Geometry}

THUNDER actuators are typically comprised of a piezoceramic wafer, a metallic backing material, hot melt adhesive layers, and an optional metallic top layer. As detailed in [4], materials commonly employed for backing layers include aluminum, stainless steel and brass while LaRC-SI is employed as the adhesive.

During the manufacturing process, the materials are placed in a vacuum bag and heated to $325^{\circ} \mathrm{C}$ under a pressure of $241.3 \mathrm{kPa}$. During the cooling process, the LaRC-SI solidifies at approximately $270^{\circ} \mathrm{C}$ and subsequent cooling produces curvature in the actuator due to differing thermal coefficients of the constituent materials. Because the Curie temperature for PZT-5A $\left(350^{\circ} \mathrm{C}\right)$ is in the proximity of the manufacturing temperature, the final step in the fabrication process is comprised of repoling the material through the application of a sustained DC voltage.

THUNDER actuators have a characteristic dome shape due to the manifestation of differing thermal properties in the PZT and backing material during the cooling process and the rotation of dipoles during repoling. In general, curvature will occur in both component directions in a rectangular actuator; however, for the models developed here, we consider actuators whose width is small as compared with the length so that motion is predominantly in one dimension. Finally, we note that the curvature in the actuators occurs only in regions covered by the piezoceramic patch and hence the end tabs remain initially straight.

The radius of curvature $R$ is calculated utilizing geometry and experimental measurements of the dome height $h$. As detailed in [4], the arclength of the tabs and PZT, denoted $t$ and $s$, the dome height and the radius of curvature are related by

$$
h=R\left[1-\cos \left(\frac{s}{2 R}\right)\right]+t \cdot \sin \left(\frac{s}{2 R}\right) .
$$

Equation (1) is a nonlinear equation in which the radius of curvature can be calculated from experimental measurements of the dome height.

\subsection{Neutral Surface}

The reference surface for the displacements in the actuator is taken to be the neutral surface $z_{n s}$. To satisfy the Kirchhoff hypothesis as detailed in [1], the displacements are assumed to be linear in the thickness direction $z$. Therefore, the mechanical stress $\sigma$ and total strain $e$ in the actuator, as depicted in Figure 2a, are related by $\sigma=Y e$ and $e=\varepsilon+\kappa\left(z-z_{n s}\right)$ where $\varepsilon$ and $\kappa$ respectively denote the midsurface strain and change in curvature while $Y$ is the Young's modulus. To calculate $z_{n s}$, a force balance at equilibrium under the assumption of pure bending (i.e., $\varepsilon=0$ ) yields

$$
\int_{0}^{h} Y_{b} \kappa\left(z-z_{n s}\right) d z+\int_{h}^{h+h_{p e}} Y_{p e} \kappa\left(z-z_{n s}\right) d z=0
$$




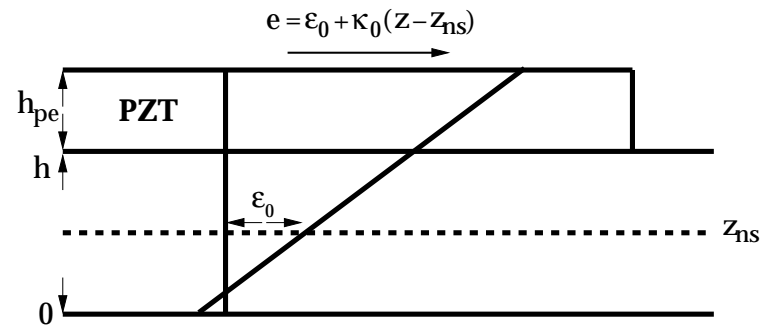

(a)

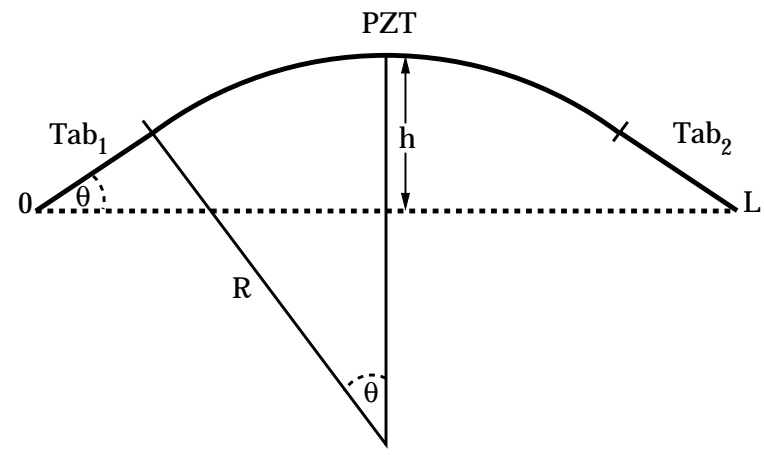

(b)

Figure 2. (a) Neutral surface of THUNDER actuator with the total strain $e$ decomposed into a pure stretching component $\varepsilon$ and a pure bending component $\kappa$; (b) Actuator geometry with flat tabs.

where $h$ and $h_{p e}$ are the thicknesses of the steel and PZT layers while $Y_{b}$ and $Y_{p e}$ are the Young's modulus of the steel and PZT layers respectively. Solving (2) for the neutral surface gives

$$
z_{n s}=\frac{Y_{b} h^{2}+Y_{p e} h_{p e}^{2}+2 Y_{p e} h_{p e} h}{2\left(Y_{p e} h_{p e}+Y_{b} h\right)}
$$

It is noted that taking the limit as $h \rightarrow 0$ (i.e., no backing material) implies that $z_{n s}=\frac{h_{p e}}{2}$ (the middle of the PZT layer) and that taking the limit as $h_{p e} \rightarrow 0$ (i.e., no PZT layer) implies that $z_{n s}=\frac{h}{2}$ (the middle of the steel layer).

\section{Hysteretic Electric Field-Polarization Relationship}

In this section, we develop the hysteretic stress-strain relationships used in the displacement model. The force and moment resultants due to an applied electric field are also derived. A nonlinear electric field-polarization relationship that is used to model the hysteresis and nonlinear saturation exhibited in a PZT patch is also discussed.

\subsection{Hysteresis Model}

As detailed in [12], macroscopic models for the hysteretic electric field-polarization relation in piezoceramics are typically based on phenomenological principles, thermodynamic tenets, or energy formulations employed in concert with homogenization techniques. Preisach theory has the advantage of providing a mathematical model quantifying hysteresis when the underlying physics is poorly understood. However, the resulting models tend to have a large number of nonphysical parameters which is undesirable for parameter identification applications. Also, the original Preisach theory does not accommodate reversible effects or variable operating temperatures and the inclusion of such effects significantly diminishes the efficiency of the resulting models.

The macroscopic polarization model used in this paper is developed in [12] through a combination of free energy principles at the lattice level and stochastic homogenization techniques which accommodates transient dynamics and ensures closure of biased minor loops. The model is of sufficiently low order to facilitate real-time control implementation as analogously illustrated for magnetostrictives in [16]. Boltzmann principles are used to construct the Helmholtz energy through an internal energy balance of positive and negative dipole configurations and entropy effects. A Gibbs relation $G$ quantifying the change in the energy landscape due to an applied field is provided by the inclusion of the electrostatic work term and the necessary condition $\frac{\partial G}{\partial P}=0$ gives the local polarization. 
To establish a constitutive relation as in [13], we assume the relation between the applied voltage $V(t)$ or electric field $E(t)$ and the generated strains $e$ exhibits hysteresis and saturation nonlinearities. Furthermore, we assume the relation between the polarization $P(t)$ and strain is linear so that all nonlinear effects occur in the relationship between the field and the polarization. This leads to a hysteretic constitutive relation

$$
\widehat{\sigma}_{p e}=Y^{P} \beta P\left(E, \widehat{\sigma}_{p e}\right)
$$

where $Y^{P}$ is the Young's modulus at constant polarization values, $P\left(E, \widehat{\sigma}_{p e}\right)$ is the polarization, which is quantified in the following subsections and $\beta$ is a scaling constant. The external forces and moments $\widehat{N}$ and $\widehat{M}$ are then calculated by integrating the stress through the thickness of the actuator,

$$
\widehat{N}=\int_{h}^{h+h_{p e}} \widehat{\sigma}_{p e} \chi_{p e} d z \quad, \quad \widehat{M}=\int_{h}^{h+h_{p e}} \widehat{\sigma}_{p e}\left(z-z_{n s}\right) \chi_{p e} d z .
$$

\subsubsection{Energy Formulation}

For fixed temperatures, we employ the theory of [12] in which it is illustrated that a reasonable formulation for the Helmholtz energy is the piecewise quadradic relation

$$
\psi(P)= \begin{cases}\frac{1}{2} \eta\left(P+P_{R}\right)^{2} & , \quad P \leq-P_{I} \\ \frac{1}{2} \eta\left(P-P_{R}\right)^{2} & , \quad P \geq P_{I} \\ \frac{1}{2} \eta\left(P_{I}-P_{R}\right)\left(\frac{P^{2}}{P_{I}}-P_{R}\right) & , \quad|P|<P_{I}\end{cases}
$$

where $P_{I}$ and $P_{R}$ denote the positive inflection point and polarization at which the minimum occurs. The resulting Gibbs free energy is derived by combining the potential energy of a dipole in the field with the Helmholtz free energy throughout the lattice to yield $G=\psi-E P$.

It is illustrated in [12] that under the assumption of low thermal activation, the local average polarization $\bar{P}$, at fixed temperature, can be derived from the necessary condition that $\frac{\partial G}{\partial P}=0$. For computational efficiency, $\bar{P}$ is formulated as

$$
\bar{P}=\frac{E}{\eta}+P_{R} \Delta
$$

where $\Delta=1$ if evaluating on the upper branch of the hysteresis kernel and $\Delta=-1$ if evaluating on the lower branch.

\subsubsection{Coercive Field and Effective Fields}

Nonuniformities in the lattice structure of the PZT produce a distribution of Helmholtz and Gibbs free energy profiles which can be manifested as variations in the local coercive field and local remanent polarization and can produce differing saturation behaviour after dipole switching. Other variations can be produced by stress nonhomogeneities, nonuniform lattice orientations across grain boundaries and crystalline anisotropies.

To incorporate variability in the lattice structure in the piecewise quadratic Helmholtz model (6), we consider the coercive field $E_{c}$ to be manifestations of an underlying distribution of the form

$$
f\left(E_{c}\right)=\exp \left[-\left(\frac{\ln \left(E_{c} / \bar{E}_{c}\right)}{2 c}\right)^{2}\right],
$$

rather than fixed values which is typically assumed for single crystals having a uniform lattice structure.

In creating a macroscopic model for the polarization, we also consider the effective fields in the material. As detailed in [12], an applied field in a ferroelectric is augmented by fields generated by neighboring dipoles which produce non-homogeneous effective fields in the material. This produces variations in the applied field that can significantly alter the resulting polarization. To incorporate these variations, we consider the effective field to be normally distributed about the applied field. 
The introduction of variations in the effective field produces domain switching in advance of the remanence point in accordance with observations from experimental data. The complete macroscopic polarization model, as derived in [12], for nonhomogeneous, polycrystalline materials with variable effective fields is given by

$$
[P(E)](t)=\int_{0}^{\infty} \int_{-\infty}^{\infty}\left[\bar{P}\left(E+\varepsilon ; E_{c}\right)\right](t) e^{-(\varepsilon)^{2} / b} e^{-\left[\ln \left(E_{c} / \bar{E}_{c}\right) / 2 c\right]^{2}} d \varepsilon d E_{c}
$$

where $\bar{P}$ is defined by (7). Note that (9) does not incorporate ferroelastic coupling so this formulation of the polarization model should be restricted to low frequency drive regimes.

\section{THUNDER Model Development}

In this section, we develop models which quantify the displacements produced in the actuators due to the input of voltages to the PZT or externally applied loads. Curved and flat beam equations are coupled together through appropriate interface conditions to yield a physically accurate representation of the THUNDER actuator. The Donnell-Mushtari version of thin shell theory is used to obtain the strain-displacement relations when describing the deformation of the middle surface. A hysteretic stress-strain relation derived from the nonlinear electric field-polariztion relation 9 is employed to describe the electromechanical coupling in the PZT. Since THUNDER actuators are used in various configurations, several boundary conditions are introduced and their numerical implementation is discussed. To facilitate numerical implementation of the model, a corresponding weak formulation is derived and employed in the examples.

\subsection{Displacement Model}

The displacement model is derived under the assumption that the actuator region covered by the patch is curved while the tabs are initially straight. To specify the geometry, we let $\gamma$ denote the arclength with $\gamma=0$ at the left end of the actuator as depicted in Figure 3. We assume that the tabs have equal length and that the portion covered by the piezoceramic patch has arclength $\left(\gamma_{2}-\gamma_{1}\right)$. The region covered by the patch is denoted by $\chi_{p e}(\gamma)=\left[\gamma_{1}, \gamma_{2}\right]$ where

$$
\chi_{p e}(\gamma)= \begin{cases}1 & , \gamma_{1} \leq \gamma \leq \gamma_{2} \\ 0 & , \text { otherwise }\end{cases}
$$

while $[0, L]$ again denotes the support for the full actuator. In the tabs, we have $\gamma=x$ whereas $\gamma=R \theta$ for the curved portion of the actuator. The radius of curvature is denoted by $R$ and calculated from dome height measurements using relation (1). The backing material is assumed to have width $b$, thickness $h$, density $\rho_{b}$ and Young's modulus $Y_{b}$ while the corresponding material parameters for the PZT layer are $b_{p e}, h_{p e}, \rho_{p e}$ and $Y_{p e}$, respectively. The longitudinal and transverse displacements, which are coupled due to the curvature, are respectively denoted by $v$ and $w$.

Under the assumptions of linear displacements, negligible rotational effects and shear deformation, and linear internal stress-strain relations, the motion of the actuator can be described by the motion of the neutral

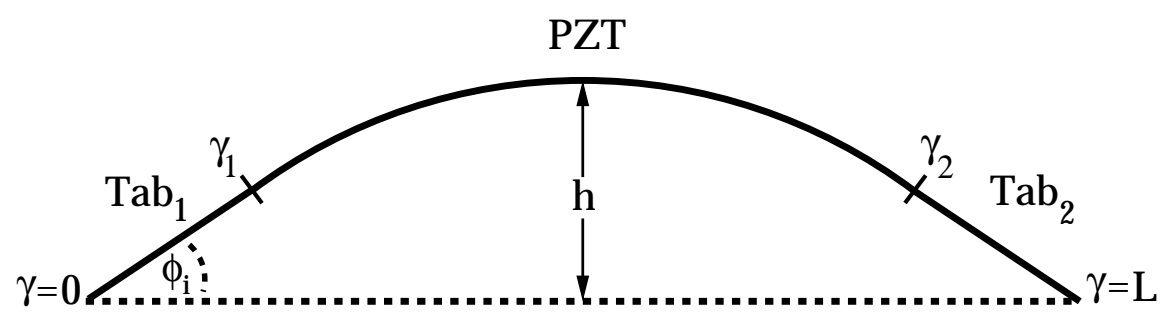

Figure 3. Geometry and arclength of the composite actuator. 
surface. A force and moment balancing under these assumptions for the curved portion of the actuator yields the dynamic equations

$$
\begin{aligned}
& \rho(\gamma) \frac{\partial^{2} v}{\partial t^{2}}-\frac{\partial N_{\gamma}}{\partial \gamma}=-\frac{\partial \widehat{N}_{\gamma}}{\partial \gamma}, \\
& \rho(\gamma) \frac{\partial^{2} w}{\partial t^{2}}-\frac{\partial^{2} M_{\gamma}}{\partial \gamma^{2}}+\frac{1}{R} N_{\gamma}=\hat{q}_{n}-\frac{\partial^{2} \widehat{M}_{\gamma}}{\partial \gamma^{2}}
\end{aligned}
$$

where $\rho(\gamma)=\rho_{b} h+\rho_{p e} h_{p e} \chi_{p e}$.

Here $N_{\gamma}$ and $M_{\gamma}$ denote the internal force and moment resultants while $\widehat{N}_{\gamma}$ and $\widehat{M}_{\gamma}$ represent the external forces and moments generated by applied voltages to the patches, as calculated in (5). Finally, $\hat{q}_{n}$ denotes applied normal loads to the actuator. As detailed in [1], the modeling equations (11) are consistent with the restriction of modified Donnell-Mushtari shell equations to the actuator geometry.

The internal resultants incorporate the material properties of the backing material and PZT. They are computed by integrating the stress through the thickness of the actuator which yields

$$
\begin{aligned}
N_{\gamma} & =\left[Y_{b} h+Y_{p e} h_{p e} \chi_{p e}(\gamma)\right] \cdot \varepsilon_{\gamma}+\left[Y_{b} h a_{1}+Y_{p e} h_{p e} a_{2} \chi_{p e}(\gamma)\right] \cdot \kappa_{\gamma} \\
& +\left[c h+c_{b} p e h_{p e} \chi_{p e}(\gamma)\right] \cdot \dot{\varepsilon}_{\gamma}+\left[c_{b} h a_{1}+c_{p e} h_{p e} a_{2} \chi_{p e}(\gamma)\right] \cdot \dot{\kappa}_{\gamma}, \\
M_{\gamma} & =\left[Y_{b} h a_{1}+Y_{p e} h_{p e} a_{2} \chi_{p e}(\gamma)\right] \cdot \varepsilon_{\gamma}+\left[Y_{b} h a_{3}+Y_{p e} h_{p e} a_{4} \chi_{p e}(\gamma)\right] \cdot \kappa_{\gamma} \\
& +\left[c_{b} h a_{1}+c_{p e} h_{p e} a_{2} \chi_{p e}(\gamma)\right] \cdot \dot{\varepsilon}_{\gamma}+\left[c_{b} h a_{3}+c_{p e} h_{p e} a_{4} \chi_{p e}(\gamma)\right] \cdot \dot{\kappa}_{\gamma}
\end{aligned}
$$

where $c_{b}$ and $c_{p e}$ are Kelvin Voight damping coefficients of the Steel and PZT layers and $\left\{a_{1}, a_{2}, a_{3}, a_{4}\right\}$ are defined by

$$
\begin{array}{cc}
a_{1}=\left(\frac{h}{2}-z_{n s}\right) \quad & a_{2}=\left(\frac{h_{p e}}{2}+h-z_{n s}\right) \\
a_{3}=\left(\frac{h^{2}}{3}-z_{n s}^{2}-h z_{n s}\right) \quad, & a_{4}=\left(\frac{h_{p e}^{2}}{3}+h^{2}+h_{p e} h+z_{n s}^{2}-2 z_{n s}-z_{n s} h_{p e}\right) .
\end{array}
$$

In the curved portion of the geometry, the midsurface strains and changes in curvature are

$$
\varepsilon_{\gamma}=\left(\frac{\partial v}{\partial \gamma}+\frac{w}{R}\right) \quad, \quad \kappa_{\gamma}=-\left(\frac{\partial^{2} w}{\partial \gamma^{2}}\right)
$$

The equations of motion (11) are indeed coupled by (14), since the PZT structure is initially curved. It is noted that the displacement equations (11) decouple as $R \rightarrow \infty$.

Since the tabs have an infinite radius of curvature, this yields the uncoupled relations

$$
\rho_{b} h \frac{\partial^{2} v}{\partial t^{2}}-\frac{\partial N_{x}}{\partial x}=-\frac{\partial \widehat{N}_{x}}{\partial x} \quad, \quad \rho_{b} h \frac{\partial^{2} w}{\partial t^{2}}-\frac{\partial^{2} M_{x}}{\partial x^{2}}=\hat{q}_{n}-\frac{\partial^{2} \widehat{M}_{x}}{\partial x^{2}}
$$

where the resultants are given by

$$
N_{x}=Y_{b} h \frac{\partial v}{\partial x}+c_{b} h \frac{\partial \dot{v}}{\partial x} \quad, \quad M_{x}=-\frac{Y_{b} h^{3}}{12} \frac{\partial^{2} w}{\partial x^{2}}-\frac{c_{b} h^{3}}{12} \frac{\partial^{2} \dot{w}}{\partial x^{2}} \quad, \quad \widehat{N}_{x}=0 \quad, \quad \widehat{M}_{x}=0 .
$$

Note that $\widehat{N}_{x}$ and $\widehat{M}_{x}$ are zero since there is no PZT in the tabular regions. Finally, the displacements and slopes at the interfaces $\gamma_{1}$ and $\gamma_{2}$ are coupled through the interface constraints

$$
\begin{array}{lll}
\lim _{\gamma \rightarrow \gamma_{1}^{-}} v(t, \gamma)=\lim _{\gamma \rightarrow \gamma_{1}^{+}} v(t, \gamma) & , & \lim _{\gamma \rightarrow \gamma_{2}^{-}} v(t, \gamma)=\lim _{\gamma \rightarrow \gamma_{2}^{+}} v(t, \gamma) \\
\lim _{\gamma \rightarrow \gamma_{1}^{-}} w(t, \gamma)=\lim _{\gamma \rightarrow \gamma_{1}^{+}} w(t, \gamma) & , & \lim _{\gamma \rightarrow \gamma_{2}^{-}} w(t, \gamma)=\lim _{\gamma \rightarrow \gamma_{2}^{+}} w(t, \gamma) \\
\lim _{\gamma \rightarrow \gamma_{1}^{-}} \frac{\partial w}{\partial \gamma}(t, \gamma)=\lim _{\gamma \rightarrow \gamma_{1}^{+}} \frac{\partial w}{\partial \gamma}(t, \gamma) \quad, & \lim _{\gamma \rightarrow \gamma_{2}^{-}} \frac{\partial w}{\partial \gamma}(t, \gamma)=\lim _{\gamma \rightarrow \gamma_{2}^{+}} \frac{\partial w}{\partial \gamma}(t, \gamma) .
\end{array}
$$




\subsection{Boundary Conditions}

We consider four sets of boundary conditions which model the constraints commonly employed in experiments: clamped-end, pinned-end, sliding-end and free-end conditions. These boundary conditions can be applied at either end of the beam; to simplify the discussion, we summarize them at the left end $(\gamma=0)$ and note that similar expressions hold at $\gamma=L$.

\section{(i) Clamped-End Conditions}

$$
v(t, 0)=0, \quad w(t, 0)=\frac{\partial w}{\partial \gamma}(t, 0)=0
$$

(ii) Pinned-End Conditions

$$
v(t, 0)=0, \quad w(t, 0)=M_{\gamma}(t, 0)=0
$$

(iii) Sliding-End Conditions

$$
w(t, 0)=v(t, 0) \tan \left(\phi_{i}\right), \quad M_{\gamma}(t, 0)=0, \quad N_{\gamma}(t, 0)=-Q_{\gamma}(t, 0) \tan \left(\phi_{c}\right)
$$

(iv) Free-End Conditions

$$
N_{\gamma}(t, 0)=M_{\gamma}(t, 0)=0
$$

In the sliding end condition, $Q_{\gamma}$ denotes the shear force resultant and $\phi_{i}$ and $\phi_{c}$ denote the initial angle of the actuator and the angle obtained after a load is applied (see Figure 3). It can be noted that to within a first-order approximation, $\phi_{i}$ and $\phi_{c}$ are related by the expression $\phi_{c}=\phi_{i}+\frac{\partial w}{\partial \gamma}$. Finally, for implementation purposes, it has been observed that physically reasonable results can be obtained with the approximation $Q_{\gamma}=0$ which is typically enforced in first-order shell theory. This yields the natural boundary condition $N_{\gamma}=0$ which is easily implemented.

We note that care must be exhibited when specifying boundary conditions at the left and right ends of the actuator to ensure model well-posedness. For example, the specification of free-end conditions at both $\gamma=0$ and $\gamma=L$ will yield rigid body modes and hence will not enforce unique solutions since solutions differing by a constant will be equivalent. For the experiments reported in Section 6, clamped-end conditions were enforced at $\gamma=0$ and sliding-end conditions were employed at $\gamma=L$.

\subsection{Weak Formulation}

To accommodate the discontinuities due to the patch and to reduce smoothness requirements on the basis functions employed for numerical implementation, we consider a corresponding weak form of the modeling system. The state space is taken to be the square-integrable function space $X=L^{2}(\Omega) \times L^{2}(\Omega)$ where $\Omega$ is the support of the actuator. The test functions depend upon the boundary conditions under consideration. For clamped, pinned, or sliding-end boundary conditions at $\gamma=0$ and free-end conditions at $\gamma=L$, we respectively employ the spaces

$$
\begin{aligned}
& V=\left\{(\varphi, \psi) \in H^{1} \times H^{2} \mid \varphi(0)=0, \psi(0)=\psi^{\prime}(0)=0\right\} \\
& V=\left\{(\varphi, \psi) \in H^{1} \times H^{2} \mid \varphi(0)=0, \psi(0)=0\right\} \\
& V=\left\{(\varphi, \psi) \in H^{1} \times H^{2} \mid \psi(0)=\varphi(0) \tan \left(\phi_{i}\right)\right\} .
\end{aligned}
$$

We note that the constraints $M_{\gamma}(L)=N_{\gamma}(L)=0$ are natural boundary conditions which do not require any restriction of the underlying Sobolev spaces. Analogous definitions are employed when considering other 
combinations of boundary conditions. It is noted that the first derivatives of the longitudinal test functions and the second derivatives of the transverse test functions may not exist at the interface points $\gamma_{1}$ and $\gamma_{2}$.

A weak form of the displacement model (11) is then:

Find $(v, w)$ such that

$$
\begin{aligned}
& \int_{0}^{L}\left\{\rho(\gamma) \frac{\partial^{2} v}{\partial t^{2}} \varphi+N_{\gamma} \frac{\partial \varphi}{\partial \gamma}-\widehat{N}_{\gamma} \frac{\partial \varphi}{\partial \gamma}\right\} d \gamma=0 \\
& \int_{0}^{L}\left\{\rho(\gamma) \frac{\partial^{2} w}{\partial t^{2}} \psi-M_{\gamma} \frac{\partial^{2} \psi}{\partial \gamma^{2}}+\frac{1}{R} N_{\gamma} \psi-\hat{q}_{n} \psi+\widehat{M}_{\gamma} \frac{\partial^{2} \psi}{\partial \gamma^{2}}\right\} d \gamma=0
\end{aligned}
$$

for all $(\varphi, \psi)$ in the appropriate space $V$.

\section{Numerical Implementation Techniques}

To find the solution of (23), we use a Galerkin finite element method with basis functions chosen to satisfy smoothness requirements as well as boundary and interface conditions. Reference elements and an ordering of the unknowns are chosen to reduce the computational cost. Transition elements are used at the material interfaces to reduce the order of the discretized model and still maintain numerical accuracy. Gaussian quadrature is used for the resulting integrals of the basis functions and a Crank-Nicholson scheme is used to solve the resulting first-order system of differential equations.

\subsection{Transition Elements}

Transition elements can be used to reduce the number of degrees of freedom from one element to another. The one dimensional elements presented here take the function and the derivative value on one side of the element and reduce it to just the function value on the other side. This element is useful in taking a cubic Hermite element, where both the function and the derivative values are continuous and reducing it to just the function value. Figure $4 \mathrm{~b}$ illustrates how two cubic Hermite elements can be joined together so that only the function value is continuous across the interface.

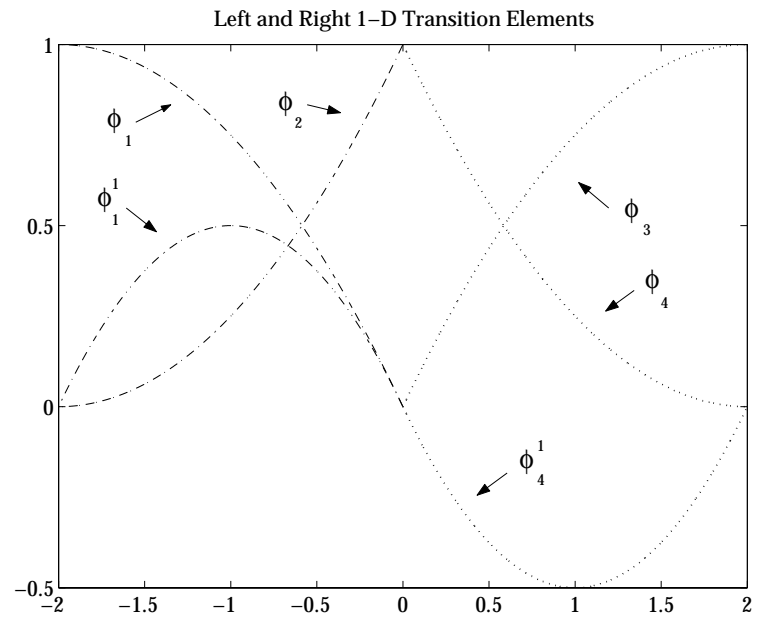

(a)

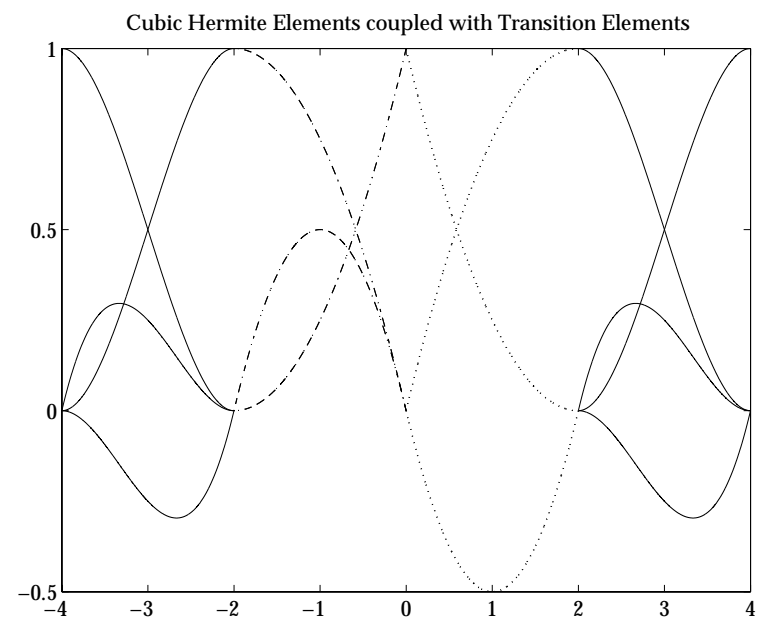

(b)

Figure 4. (a) Left (-•) and right (..) transition elements reducing the degrees of freedom from 2 on the boundary to 1 in the interior. (b) Left (-.) and right (..) transition elements adjoining two cubic Hermite elements (-). Only the function value is continuous across $\xi=0$. 
This technique is well suited to physical problems involving two homogeneous materials joined at an interface. Inside each homogeneous material, a function could be continuous and have a continuous first derivative. However, at the interface, only the function itself is required to be continuous. The use of cubic Hermite basis functions will give the $C^{1}(\Omega)$ continuity in the interior of each region, while transition elements on either side of the interface would force only the function to be continuous across the interface.

The transition elements in Figure 4a, as derived in [11], consists of the right and left elements

$$
\begin{aligned}
& \phi_{1}=\frac{3}{4}-\frac{1}{2} \xi-\frac{1}{4} \xi^{2}, \quad \phi_{1}^{1}=\frac{1}{2}-\frac{1}{2} \xi^{2}, \quad \phi_{2}=\frac{1}{4}+\frac{1}{2} \xi+\frac{1}{4} \xi^{2} \\
& \phi_{3}=\frac{1}{4}-\frac{1}{2} \xi+\frac{1}{4} \xi^{2}, \quad \phi_{4}=\frac{3}{4}+\frac{1}{2} \xi-\frac{1}{4} \xi^{2}, \quad \phi_{4}^{1}=-\frac{1}{2}+\frac{1}{2} \xi^{2}
\end{aligned}
$$

where $\xi \in[-1,1]$.

\subsection{Finite Element Method}

We begin by dividing the THUNDER actuator into 3 regions. The first and third regions $\left[0, \gamma_{1}\right]$ and $\left[\gamma_{2}, L\right]$, are the straight steel tabs which do not support the piezoceramic while the curved second region $\left[\gamma_{1}, \gamma_{2}\right]$, consists of the PZT and the steel backing as depicted in Figure 5a. Each region is uniformly partitioned into $N_{i},(i=1,2,3)$ elements for a total of $N=N_{1}+N_{2}+N_{3}$ elements across the entire actuator with the nodes labeled as $\theta_{i},(i=0, \cdots, N)$ as in Figure $5 \mathrm{~b}$. The set of nodes that correspond to the interface elements of the 3 regions (i.e., nodes of elements $N_{1}, N_{1}+1, N_{1}+N_{2}, N_{1}+N_{2}+1$ ) are labeled $\partial \Omega_{N}$ while the remaining nodes away from the material discontinuities are in the set denoted by $\Omega_{N}$. These sets will be used to differentiate between transition elements and cubic Hermite elements when we define the finite element space.

The finite element space $V_{N}$ considered consists of cubic Hermite and transition elements that satisfy boundary and interface conditions. Left and right transition elements $\phi_{k}$ are defined by (24) while the cubic Hermite shape functions satisfy

$$
\psi_{j}^{0}\left(\theta_{i}\right)=\delta_{i j}, \quad \psi_{j}^{1}\left(\theta_{i}\right)=0, \quad \frac{d \psi_{j}^{0}}{d \theta}\left(\theta_{i}\right)=0, \quad \frac{d \psi_{j}^{1}}{d \theta}\left(\theta_{i}\right)=\delta_{i j}
$$

where $\delta_{i j}$ is the Kronecker delta. The $i^{t h}$ basis functions also vanish outside the interval $\left[\theta_{i-1}, \theta_{i+1}\right]$. In the space $V_{N}$, the longitudinal displacement $v\left(\theta_{l}\right)$ is a linear combination of the transition and cubic Hermite basis functions which we shall call $\varphi_{i}$ and the transverse displacement $w\left(\theta_{l}\right)$ is a linear combination of the cubic Hermite basis functions $\psi_{j}$,

$$
\begin{gathered}
v\left(\theta_{l}\right)=\sum_{i=0}^{N} v_{i} \varphi_{i}\left(\theta_{l}\right)=\sum_{i \in \Omega_{N}} v_{i} \psi_{i}^{0}\left(\theta_{l}\right)+\sum_{i \in \Omega_{N}} v_{i}^{\prime} \psi_{i}^{1}\left(\theta_{l}\right)+\sum_{i \in \partial \Omega_{N}} v_{i} \phi_{i}\left(\theta_{l}\right) \\
w\left(\theta_{l}\right)=\sum_{j=0}^{N} w_{j} \psi_{j}^{0}\left(\theta_{l}\right)+\sum_{j=0}^{N} w_{j}^{\prime} \psi_{j}^{1}\left(\theta_{l}\right)
\end{gathered}
$$

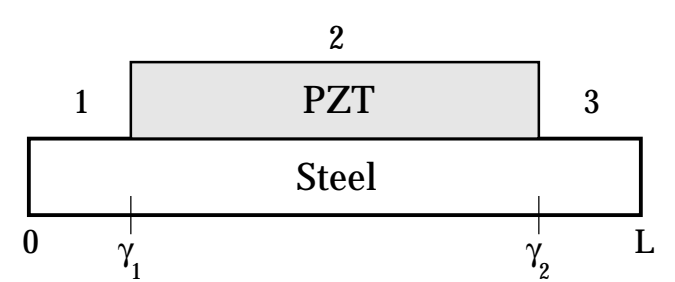

(a)

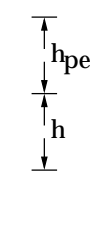

h

(a)

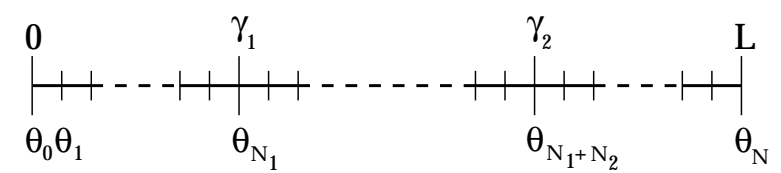

(b)

Figure 5. (a) Partition of THUNDER actuator; (b) Coordinate system 
where $\left\{v_{i}, v_{i}^{\prime}, w_{i}, w_{i}^{\prime}\right\}$ represent the function and derivative values of $v\left(\theta_{l}\right)$ and $w\left(\theta_{l}\right)$ at the $i^{t h}$ node (i.e., $v_{i}=$ $\left.v\left(\theta_{i}\right)\right)$.

The essential boundary conditions in (22) are enforced by removing or forming linear combinations (in the case of the sliding condition) of the boundary basis functions $\left\{\varphi_{0}, \psi_{0}^{0}, \psi_{0}^{1}, \varphi_{N}, \psi_{N}^{0}, \psi_{N}^{1}\right\}$. The interface conditions are imposed by formulating the model with respect to arclength in each region and having only 1 node at the interfaces. This enforces the appropriate function and derivative continuity conditions across the interfaces.

The basis functions together with the boundary conditions define the finite element space $V_{N}$. A linear system of equations is obtained by considering a discretization of the weak formulation of the model (23) with basis functions employed as test functions. This yields a linear system expressed as the following matrix system,

$$
\begin{aligned}
& A \ddot{v}+B \dot{v}+C \dot{w}+D v+E w=\widehat{N} \\
& F \ddot{w}+G \dot{v}+H \dot{w}+I v+J w=\widehat{M}
\end{aligned}
$$

where the matrices $A, \cdots, J$ consist of discretized integrals of the basis functions using Gaussian quadrature so that they are computed to machine accuracy. The coefficients of $v$ and $w$ as a vector of unknowns are ordered such that the value of the function and of the derivative at the nodes are adjacent. This reduces the number of bands in the matrices $A, \cdots, J$ which lowers the cost in solving the linear system, as compared to a complete ordering of function values at the nodes followed by derivative values. Since the partition in each region is uniform, the use of reference elements reduces the cost of forming the matrices by only requiring 1 calculation of the basis function integrals per region. To solve these second order equations, we consider the method of reducing the equations to a first order system. This has the advantage of utilizing first order ODE solvers and is of standard form for linear control theory. The disadvantage is that the size of the system is doubled and the mass matrices $A$ and $F$ must be inverted and stored, thus losing the sparseness of the problem.

The first order system will be of the form $\dot{x}=\tilde{A} x+b$ where $x=\left[v_{0}, v_{0}^{\prime}, \cdots, v_{N}, v_{N}^{\prime}, w_{0}, w_{0}^{\prime}, \cdots, w_{N}, w_{N}^{\prime}\right]^{T}$ denotes a vector of the unknown coefficients given in (26). This can be integrated in time by using backward Euler or Crank-Nicholson (Trapezoid) rather easily. The system in first order form is,

$$
\left(\begin{array}{c}
\dot{v} \\
w \\
\dot{v} \\
\dot{w}
\end{array}\right)=\left(\begin{array}{cccc}
0 & 0 & 1 & 0 \\
0 & 0 & 0 & 1 \\
-A^{-1} D & -A^{-1} E & -A^{-1} B & -A^{-1} C \\
-F^{-1} I & -F^{-1} J & -F^{-1} G & -F^{-1} H
\end{array}\right)\left(\begin{array}{c}
v \\
w \\
\dot{v} \\
\dot{w}
\end{array}\right)+\left(\begin{array}{c}
0 \\
0 \\
A^{-1} \widehat{N} \\
F^{-1} \widehat{M}
\end{array}\right) .
$$

It is noted that the steady state conditions $\dot{v}=\dot{w}=0$ yields the steady state equations of motion.

\subsection{Time Integration and Boundary Conditions}

A Crank-Nicholson scheme is used to perform the time discretization of (28). This is done by building the global matrix $\tilde{A}$ on the entire interval $[0, L]$ and approximating the integral of (28) with the trapezoid method. This leads to the system,

$$
\left(1-\frac{\Delta t}{2} \tilde{A}\right) x_{+}=\left(1+\frac{\Delta t}{2} \tilde{A}\right) x_{-}+\Delta t b
$$

which we will denote $\bar{A} x_{+}=\bar{b}$. Since the matrices $A, \cdots, J$ are constructed on the entire interval $[0, L]$, the global matrix $\bar{A}$ will have full rank. However, construction on the entire interval does not enforce all of the boundary conditions. Doing this is equivalent to free boundary conditions on both ends, which is not well-posed. To enforce a clamped boundary condition, the appropriate row in the global matrix $\bar{A}$ must be zeroed out with a 1 placed on the diagonal to keep the matrix from going singular. The appropriate node in the right hand side $\bar{b}$ must also be set to the boundary condition value. As an example, consider the PZT fixed at the left end. This means that the node $x_{0}$ is a boundary node. To make sure the displacements are zero at that node, we shall set the value of $v_{0}$ and $w_{0}$ to zero. This is done by zeroing out the first row of $\bar{A}$ and setting $a_{(1,1)}$ to 1 and zeroing out row $N+1$ in $\bar{A}$ (since the length of $v$ is $N$, the value of $w$ at the left boundary node is in row $N+1$ in the vector $x)$ and setting $a_{((N+1),(N+1))}$ to 1 . Finally, $\bar{b}_{1}$ and $\bar{b}_{N+1}$ are set to zero. 


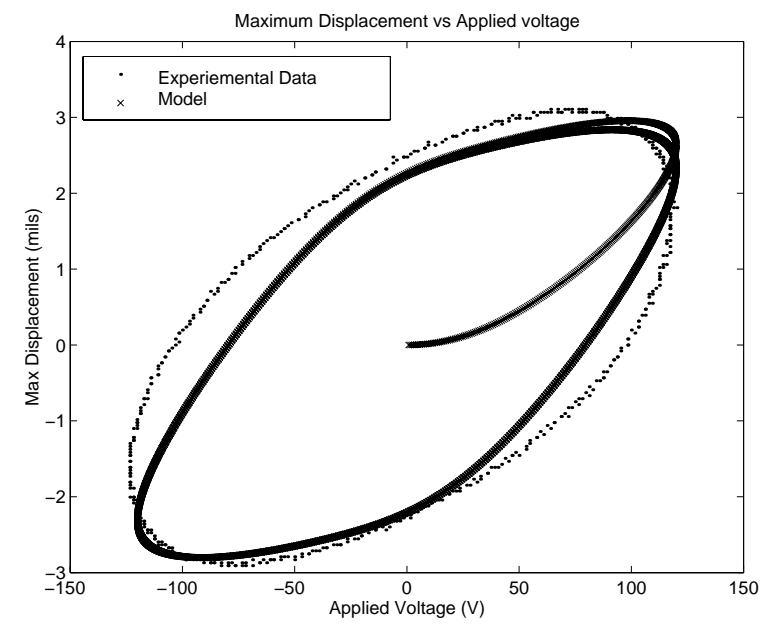

Figure 6. Displacement model $(\times)$ vs Experimental data $(\cdot)$

\section{Experimental Validation}

To illustrate the performance of the displacement model presented in Section 4, we consider an actuator having clamped-end conditions at the left edge $(\gamma=0)$ and sliding-end conditions at the right edge $(\gamma=L)$. The thickness of the PZT patch is 8 mils while the thickness of the steel backing is 10 mils. The LaRC-SI glue layer is $1 \mathrm{mil}$ in thickness and its contribution to the displacement model is ignored. The actuator is driven with 120 Volts at $1 \mathrm{~Hz}$ to minimize the inertial effects. The displacement data is taken by measuring the maximum vertical displacement of the actuator from the zero voltage rest position. The numerical data is taken by interpolating the results from the discretized model and calculating the maximum displacement.

The models relation between the input voltage and strains generated in the THUNDER transducer is compared with experimental data in Figure 6. To illustrate transient behavior quantified by the model, we start with an initial voltage of $0 \mathrm{~V}$ and apply five periodic input cycles. By the second cycle, the modeled dynamics have settled into the periodic cycle exhibited by the steady state data. The parameters $P_{R}=0.03, \eta=0.25$, $\bar{E}_{c}=1.9899 \times 10^{-3}, c=1.5188, b=2.2407 \times 10^{-4}, c_{2}=41.366, \beta=1.152 \times 10^{-5}, Y_{p e}=1.77 \times 10^{11}$, $Y_{b}=1.73 \times 10^{11}, c_{p e}=1.0 \times 10^{9}$ and $c_{b}=2.00 \times 10^{10}$ were obtained through a least squares fit to the data.

\section{Conclusions}

The model described here provides a technique for quantifying the displacements in a THUNDER actuator generated by applied voltages by utilizing a hysteretic electric field-polarization relationship. The hysteresis and saturation nonlinearities were modeled with a free energy approach while Newtonian principles were used to derive PDE models characterizing displacements for a variety of boundary conditions and exogenous loads. The validity of the displacement model was illustrated through comparison with experimental data.

It should be noted that dynamic elastic effects were not used in deriving the E-P relation and therefore the model should be restricted to low drive regimes. The inclusion of such terms and other linear dielectric loss terms is currently under investigation. 


\section{Acknowledgments}

This research was supported by NASA Grant NAG-1-01041.

\section{References}

[1] H.T. Banks, R.C. Smith and Y. Wang, Smart Material Structures: Modeling, Estimation and Control, Masson/John Wiley, Paris/Chichester, 1996.

[2] B.A. Boley and J.H. Weiner, Theory of Thermal Stresses, John Wiley and sons, New York, 1960.

[3] R. Wieman, R.C. Smith, T. Kackley, Z. Ounaies and J. Bernd, "Displacement models for THUNDER actuators having general loads and boundary conditions", Proceedings of the SPIE, Smart Structures and Materials 2001, 4326, pp. 252-263, 2001.

[4] M. Capozzoli, J. Gopalakrishnan, K. Hogan, J. Massad, T. Tokarchik, S. Wilmarth, H.T. Banks, K.M. Mossi and R.C. Smith, "Modeling Aspects Concerning THUNDER Actuators", Proceedings of the SPIE, Smart Structures and Materials 1999, 3667, pp. 719-727, 1999.

[5] D.L. Edberg, "Control of flexible structures by applied thermal gradients", AIAA Journal, 25(6), pp. 877$883,1987$.

[6] A. Hamamoto and M.W. Hyer, "Nonlinear temperature-curvature relationships for unsymmetric graphite epoxy laminates", International Journal of Solids and Structures, 23(7), pp. 919-935, 1987.

[7] M.W. Hyer, "The room-temperature shapes of four-layer unsymmetric cross-ply laminates", Journal of Composite Materials, 16(4), pp. 318-340, 1981.

[8] M.W. Hyer and A. Jilani, "Predicting the deformation characteristics of rectangular unsymmetrically laminated piezoelectric materials", Smart Materials and Structures, 7, pp. 1-8, 1998.

[9] B. Jaffe, W.R. Cook, Jr. and H. Jaffe, Piezoelectric Ceramics, Academic Press, New York, 1971.

[10] A.J. Moulson and J.M. Herbert, Electroceramics, Chapman and Hall, New York, 1990.

[11] L.R. Ram-Mohan, Finite Element and Boundary Element Applications in Quantum Mechanics, Oxford, 2002.

[12] R.C. Smith, S. Seelecke, Z. Ounaies and J. Smith, "A free energy model for hysteresis in ferroelectric materials", CRSC Technical Report CRSC-TR03-01; Journal of Intelligent Material Systems and Structures, submitted.

[13] R.C. Smith and M.V. Salapaka, "Model development for the positioning mechanisms in an atomic force microscope", International Series of Numerical Mathematics, 142, pp. 249-269, 2002.

[14] R.C. Smith and C.L. Hom, "Domain wall theory for ferroelectric hysteresis", Journal of Intelligent Material Systems and Structures, 10(3), pp. 195-213, 1999.

[15] R.C. Smith and Z. Ounaies, "A domain wall model for hysteresis in piezoelectric materials", Journal of Intelligent Material Systems and Structures, 11(1), pp. 62-79, 2000.

[16] J. Nealis and R.C. Smith, "Robust Control of Magnetostrictive Actuator" Proceedings of the SPIE, Smart Structures and Materials 2003, to appear. 